Recepción: 07 / 09 / 2016

Aceptación: 31 / 01 / 2017

Publicación: 06 / 03 / 2017

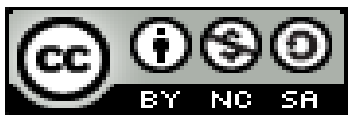

Ciencias de la salud

Artículo de investigación

\title{
Analgesia acupuntural en la actividad quirúrgica. Realidad práctica alternativa
}

Acupunctural analgesia in surgical activity. Alternative practical reality

Analgesia acupuntura na atividade cirúrgica. realidade prática alternativa

Haydeé Ramos Portuondo haydee@hospclin.scu.sld.cu

Mariuska Arias Chacón ${ }^{\mathrm{II}}$
mariuska@hospclin.scu.sld.cu
Lázara de la FéBatista ${ }^{\mathrm{III}}$
Lazaradelafe@gamil.com
María Caridad Ogra Álvarez ${ }^{\mathrm{IV}}$
ogra@ sierra.scu.sld.cu

Correspondencia: haydee@ hospclin.scu.sld.cu

\footnotetext{
I Doctora, Maestría en Ciencias, Hospital Juan B. Zayas Alfonso. Universidad de Ciencias Médicas, Santiago de Cuba, Cuba.

${ }^{\text {II }}$ Doctora, Hospital Juan B. Zayas Alfonso. Universidad de Ciencias Médicas, Santiago de Cuba, Cuba.

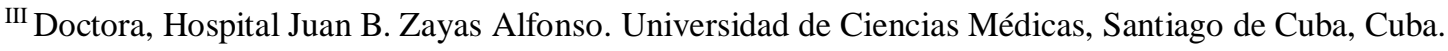

${ }^{\text {IV }}$ Doctora, Hospital Militar Joaquín Castillo Duany, Santiago de Cuba, Cuba.
} 


\title{
Resumen
}

La acupuntura es un método de tratamiento basado en la estimulación neural periférica, la cual actúa en tres niveles del sistema nervioso central: espinal, tronco encefálico y diencéfalo, en los que se involucran núcleos cerebrales (reticular del bulbo y magno de rafe), áreas del sistema límbico, neurotransmisores como serotonina, noradrenalina y acetilcolina que favorecen la liberación de neuropéptidos como las encefalinas y endorfinas las que juega un papel fundamental en la modulación de la transmisión del estímulo doloroso, lo que permite elevar el umbral del dolor; el cual constituye la base de la analgesia quirúrgica acupuntural, teniendo en cuenta las principales teorías occidentales que tratan de explicar los mecanismos de supresión de dolor se abordan las bases neurofisiológicas de esta técnica milenaria.

Palabras clave: analgesia acupuntural; medicina tradicional china; transmisión del dolor.

\begin{abstract}
Acupuncture is a treatment method based on peripheral neural stimulation, which acts on three levels of the central nervous system: spinal, brain stem and diencephalon, involving brain nuclei (reticular bulb and magne of raphe), areas of the limbic system, neurotransmitters such as serotonin, noradrenaline and acetylcholine, favoring the release of neuropeptides such as enkephalins and endorphins, which plays a key role in modulating the transmission of painful stimuli, thus raising the pain threshold; Which is the basis of acupuncture surgical analgesia, taking into account the main western theories that try to explain the mechanisms of pain suppression, the neurophysiological bases of this millenarian technique are approached.
\end{abstract}

Key words: acupuncture analgesia; chinese traditional medicine; transmission of pain.

\section{Resumo}

A acupuntura é um método de tratamento baseado na estimulação neural periférica, que opera em três níveis do sistema nervoso central: espinhal, tronco cerebral e diencéfalo, nos quais estão envolvidos núcleos cerebrais (lâmpada reticular e rafe magnus), áreas o sistema límbico, neurotransmissores, como a serotonina, norepinefrina e acetilcolina, que favorecem a libertação de neuropéptidos, tais como encefalinas e endorfinas, que desempenha um papel chave na modulação da transmissão de estímulos dolorosos, que pode elevar o limiar da dor; que é a base da analgesia acupuntura cirúrgica, tendo em conta as principais teorias ocidentais que tentam 
explicar os mecanismos de supressão da dor bases neurofisiológicas desta técnica antiga são discutidos.

Palavras chave: analgesia acupuntural; medicina tradicional chinesa; a transmissão da dor.

\section{Introducción}

Desde hace varios miles de años la humanidad conoce una técnica denominada acupuntura que ha soportado los reclamos de la comunidad científica internacional, que forma parte de un sistema médico tradicional reconocido por la Organización Mundial de la Salud (OMS), cuyo principal objetivo se ha relacionado en Occidente casi siempre con su capacidad para suprimir el dolor.

En las civilizaciones occidentales el dolor agudo ha sido siempre tratado con fármacos analgésicos; sin embargo, en el Oriente generalmente se han empleado terapéuticas de estimulación, entre las que se encuentra la acupuntura. Esta técnica se viene utilizando desde entonces para aliviar todo tipo de dolores. ${ }^{1,7}$

Desde su introducción en Europa a fines del siglo XVII, la acupuntura ha tenido diversa aceptación en el mundo occidental. Períodos de enorme interés han sido seguidos de otros de casi completo rechazo. Las razones residen en que sus ventajas fundamentales, la ausencia de efectos indeseables y la facilidad de su aplicación, contrastan con la incapacidad de médicos y pacientes occidentales para asimilar esta técnica. ${ }^{1,7}$

La observación de la eficacia de la acupuntura en la supresión del dolor condujo a los médicos chinos de la década de los años 50 del siglo XX al ensayo de su aplicación en heridos de guerra antes de cambiar los vendajes, con lo que demostraron que la dicha técnica prevenía la instauración del dolor a la vez que eliminaba el ya existente. La primera operación realizada por los cirujanos chinos con analgesia acupuntural fue una amigdalectomía. La Medicina Tradicional China (MTCh) y la Analgesia Quirúrgica Acupuntural (AQA) llegaron a Cuba en la década de los años 70 del pasado siglo XX, pero no tuvieron en aquellos momentos buena aceptación entre los facultativos. ${ }^{1,7}$

La AQA es un método analgésico que se basa en la elevación el umbral del dolor mediante el estímulo acupuntural, es decir, el bloqueo de la señal dolorosa que se ha de originar durante el 
acto quirúrgico y no permitir su paso a los centros nerviosos superiores, donde sería interpretada como dolor. ${ }^{8,} 11$

La más antigua referencia encontrada acerca del uso de la acupuntura como anestesia para la realización de intervenciones quirúrgicas, está relacionada con el primer médico cirujano conocido en China, Hua To (años 110-207 n.e.), que realizó laparotomías con el empleo del Mafeisan, supuesto anestésico general por vía oral, y dicen algunos que combinado con la analgesia acupuntural. La AQA, así denominada surgió en 1958 en China, donde se realizó la primera amigdalectomía con esta técnica analgésica en sustitución de la anestesia convencional. ${ }^{8,10}$

A esta primera intervención muy pronto siguieron otras sobre la cabeza, el cuello, el tórax, el abdomen y las extremidades. Hasta 1979 fueron realizadas en China aproximadamente 600.000 operaciones con analgesia acupuntural, a las que posteriormente se añadieron varios cientos más. También fuera de la República Popular China, ha sido publicado el uso exitoso de la AQA en cirugía mayor y menor en el Asia, en Europa y más tarde en América. ${ }^{12, ~ 8, ~} 10$

En Cuba, se reportó la realización de dos amigdalectomías en La Habana en 1976 y una en 1977. En 1982 dos médicos vietnamitas realizaron con analgesia acupuntural 29 intervenciones en el Hospital Militar "Dr. Luis Díaz Soto” y 20 operaciones en el Hospital Militar "Dr. Carlos J. Finlay". ${ }^{13}$

El 1 de septiembre de 1984 se realizó una colecistectomía con acupuntura; y en 1985 una herniorrafia umbilical. En 1986 otro profesor vietnamita realizó en el Hospital "Hermanos Ameijeiras" dos intervenciones de catarata y dos herniorrafias con analgesia acupuntural. ${ }^{14,15,10,12}$

En el Hospital Docente "Saturnino Lora", de Santiago de Cuba, en el año 1982 se realizó la primera intervención quirúrgica, amigdalectomía, luego de un período de espera se retoma esta técnica por los anestesiólogos del Hospital Militar Docente “Joaquín Castillo Duany”, en el año 1992, realizándose hasta la fecha un número significativo de casos en los que se incluyen, amigdalectomías, extracciones dentarias, mastoplastias, pólipos nasales, operaciones oftalmológicas, así como tiroidectomías, por lo que hemos decidido presentar un caso de esta última patología. ${ }^{14}$ 
En el hospital "Juan B. Zayas Alfonso", a partir del año 2014 se comienza a realizar intervenciones quirúrgicas en el centro oftalmológico con AQA, en pacientes con el diagnóstico de cataratas, glaucoma, pterigium y otras patologías oftalmológicas, con resultados satisfactorios en el postoperatorio. A inicios del 2016 se inicia la realización de colonoscopía con AQA, con buenos resultados y aceptación por los pacientes.

\section{Desarrollo}

- Fisiología del dolor.

El dolor se define como una respuesta individual del organismo, ante un estímulo, que provoca liberación de sustancias alógenas y neurotransmisoras, con la consiguiente activación de receptores, fibras y centros nerviosos. ${ }^{16,20}$

Existen diferentes clasificaciones del dolor, una de ellas lo clasifica en:

- Dolor rápido, vivo, intenso, agudo o punzante: Se transmite por fibras A-delta, poco mielinizadas.

- Dolor lento, urente, sordo o profundo, crónico o pulsátil: Se transmite por las fibras C, amielínicas.

- $\quad$ Estructuras que participan en la transmisión del dolor. $(21,19,20)$

- Nociceptores.

- Fibras nerviosas.

- Sistema trigeminal.

- Médula espinal: Cuerno posterior.

- Vías aferentes: Tractos espinotalámico, espinoreticular, espinomesencefálico, cervicotalámico, espinohypotalámico.

- Tálamo.

- Hipotálamo y sistema límbico.

- Corteza cerebral: Zona somatosensitiva. 


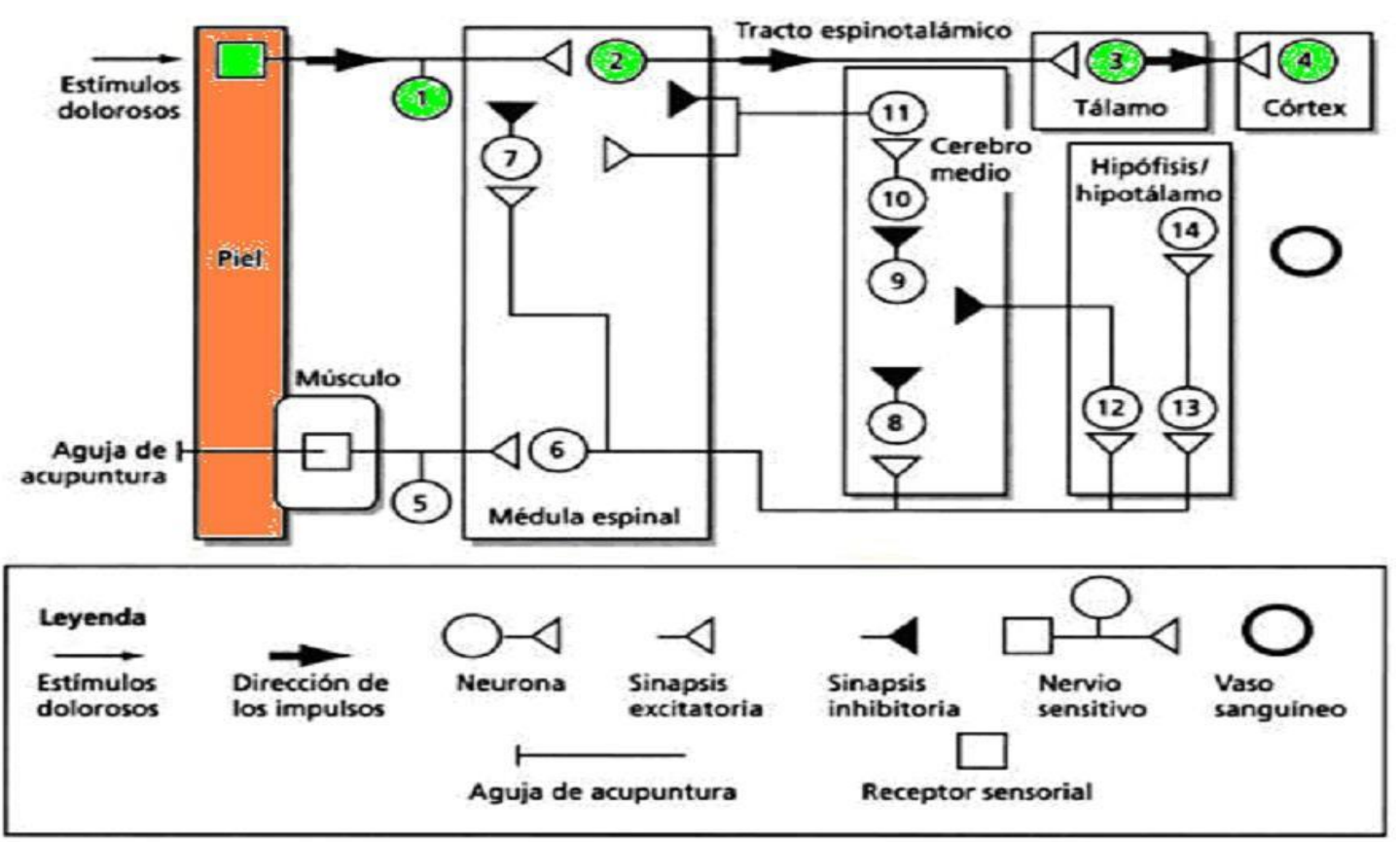

Figura 1. Estructuras que participan en la transmisión del dolor.

- $\quad$ Sustancias que excitan o inhiben a los nociceptores. 22, 26

- Bradiquinina.

- Serotonina.

- Acetilcolina.

- Prostaglandinas.

- Sustancia P.

- Endorfinas.

- Encefalinas.

- Noradrenalina.

Los péptidos opioides son todas las sustancias antagonizadas por la naloxona, que tienen como precursores polipéptidos, los que por acciones enzimáticas se van fragmentando en proencefalina, que origina la metionina y la leucina encefalina, proopiomelanocortina, origina b-endorfina, pro dinorfina, que da lugar a la dinorfina. Todos ellos se encuentran distribuidos en el sistema nervioso central y en el periférico y desempeñan un rol relevante en la modulación endógena del dolor (vías inhibidoras). ${ }^{27,}$ 29, 7, 8 
La estimulación eléctrica o la inyección de opioides en la sustancia gris periacueductal tiene como resultado una marcada analgesia debido a la gran cantidad de receptores opioides que en ella se encuentran afines a los péptidos de las familias: dinorfinas, encefalinas y b-endorfinas. La formación bulbar ventro rostro medial es la zona más sensible a la inyección de opioides y sus neuronas contienen encefalinas. En las astas posteriores se encuentran encefalinas y dinorfinas, lo que favorece la producción de analgesia. El sistema serotoninérgico tiene su sustrato anatómofuncional en el núcleo del rafe y bulbo pontino, desde donde se proyecta hacia estructuras encefálicas, corticales y la médula espinal. El sistema monoaminérgico descendente tiene como neurotransmisor a la noradrenalina y la serotonina, ambas participan activamente en la analgesia acupuntural. $30,33,28,29$

- $\quad$ Sistema de analgesia y mecanismo de acción de la Analgesia Quirúrgica Acupuntural.

Los efectos de la analgesia quirúrgica acupuntural en el sistema nervioso se logran mediante la activación de un sistema de control o inhibición del dolor llamado sistema de la analgesia, el cual está constituido por 3 elementos. ${ }^{30,33}$

1. La sustancia gris periacueductal y las áreas periventriculares del mesencéfalo (y de la parte superior de la protuberancia que rodean al acueducto de Silvio y que están contiguas a determinadas partes de los ventrículos tercero y cuarto), las neuronas de estas regiones envían sus señales a:

2. Al núcleo magno del rafe (un fino núcleo situado en la línea media de la parte baja de la protuberancia y alta del bulbo) y al núcleo reticular paragigantocelular (situado lateralmente en el bulbo). De estos núcleos las señales se trasmiten en dirección descendente hasta las columnas dorsolaterales de la médula espinal para llegar a:

3. Un complejo inhibidor del dolor situado en las astas posteriores de la médula, en las láminas II y III, donde se encuentra la sustancia gelatinosa de Rolando, que al ser excitada producen inhibición de la primera célula transmisora (célula T) que es donde se originan los haces espinotalámicos conductores del estímulo doloroso, bloqueando a este nivel la conducción de dicho estímulo al cerebro. 
El sistema de control descendente que conecta a la sustancia gris periacueductal, la formación bulbar ventro rostro medial (estación de relevo entre mesencéfalo y astas posteriores) y el segmento pontino dorsolateral y a éstos con proyecciones espino-encefálicas, cuenta con la participación de la serotonina y noradrenalina como mediadores químicos. ${ }^{34,41}$

La formación reticular bulbar ventro rostro medial que comprende el grupo posterior de los núcleos del rafe es la estructura nerviosa más importante en la modulación supraespinal acupuntural. ${ }^{34,35,38}$

Los impulsos nerviosos acupunturales ascienden vía el fascículo ventrolateral de la médula espinal, que conduce las sensaciones de dolor y temperatura hacia el cerebro, se activa el sistema antinociceptivo que comprende algunos núcleos cerebrales: caudado, arcuato, accumbens, sustancia gris periacueductal y el núcleo magno del rafe; neuromoduladores como los péptidos opioides; neurotransmisores: serotonina, noradrenalina, acetilcolina y a través de las vías inhibidoras descendentes se produce la analgesia. Los centros troncoencefálicos ricos en receptores opioiodes, la sustancia gris periacueductal y el núcleo magno del rafe, a través del funículo dorsolateral de la médula envían sus proyecciones a la médula espinal, hasta llegar a la sustancia gelatinosa de Rolando del asta posterior, bloqueando presinápticamente las fibras nociceptivas primarias o modulando interneuronas peptidérgicas y gabaérgicas, favoreciendo así la inhibición de la transmisión del estímulo doloroso. ${ }^{38,39,41}$

La estimulación del núcleo caudado puede producir analgesia por liberación de endorfinas en los receptores opioides (sitios de unión) en distintas áreas cerebrales así como en la sustancia gris periacueductal. Estudios morfológicos con diversos métodos revelaron conexiones entre el núcleo caudado y el tálamo medio; el núcleo caudado y el núcleo del rafe; el núcleo caudado y la sustancia gris periacueductal. ${ }^{39,41}$ 


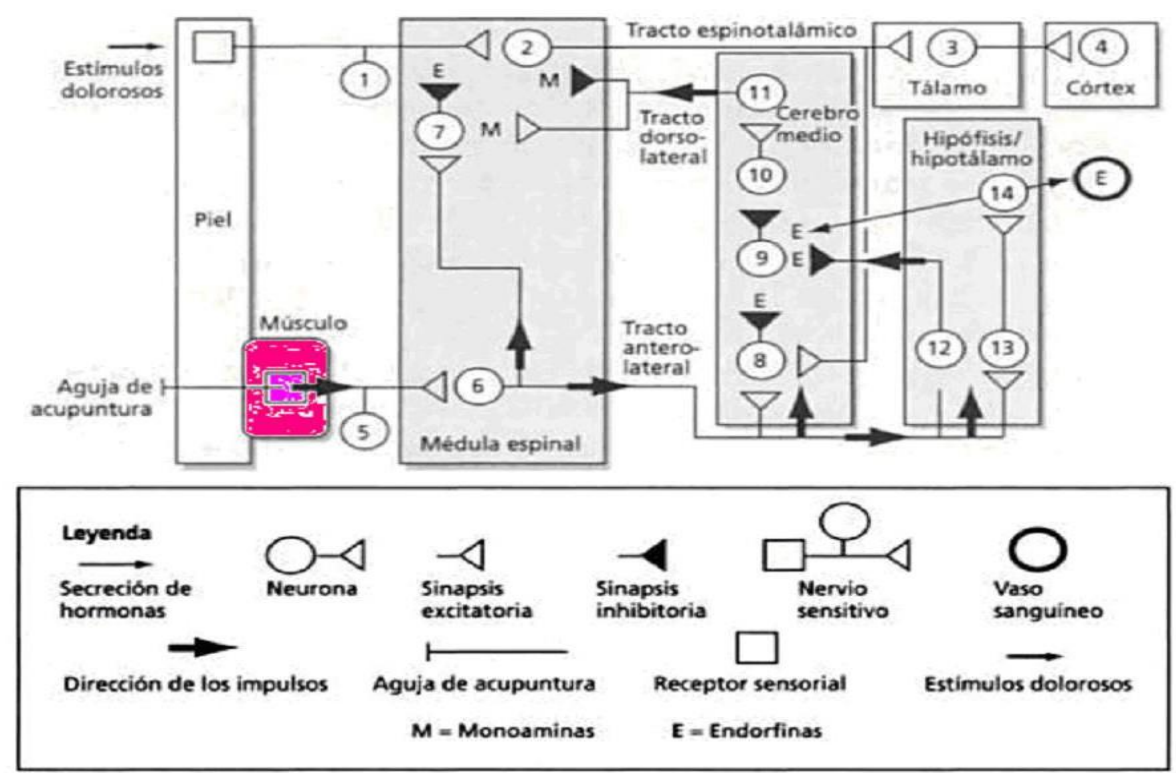

Figura 2. Mecanismo de acción de la Analgesia Quirúrgica Acupuntural.

- Ventajas de la analgesia quirúrgica acupuntural. ${ }^{42}$

1. Amplio margen de seguridad. La acupuntura no induce efectos colaterales negativos en las funciones orgánicas, y correctamente utilizada no causa accidentes. La analgesia acupuntural evita efectos secundarios indeseados en enfermos cuyo estado general es precario o padecen serios trastornos orgánicos que hacen de la anestesia convencional un gran riesgo pero en los que la intervención quirúrgica es inaplazable.

2. Pocas alteraciones fisiológicas. Durante la AQA la tensión arterial, la frecuencia cardiaca y la frecuencia respiratoria sufren modificaciones poco importantes que regresan a la normalidad entre 5 y 30 minutos posteriores a la operación. La intubación nasogástrica no es necesaria. Las grandes funciones del organismo se mantienen en estado fisiológico durante el transoperatorio y el postoperatorio.

3. El paciente coopera activamente a la operación. Durante la AQA el paciente permanece consciente, coopera con el cirujano, lo cual es importante en operaciones sobre el ojo, la parótida, el tiroides y otras, en las que se puede comprobar inmediatamente el resultado de la acción quirúrgica sin tener que esperar a que desaparezca el efecto anestésico.

4. Técnica sencilla y económica. La técnica acupuntural es simple. No necesita de equipos de gran complejidad ni se afecta por condiciones geográficas, climatológicas; requiere del empleo de 
sólo muy pequeñas dosis de algunos fármacos anestésicos locales y analgésicos. Cuando se utiliza como coadyuvante de la anestesia convencional, permite utilizar dosis menores de dichos fármacos.

5. Mejor y más rápida recuperación. Además de la supresión del dolor, la acupuntura reduce la inflamación, produce sedación, hemostasia y coadyuva al mantenimiento de la homeostasis. El postoperatorio transcurre confortablemente y la analgesia se prolonga por largo tiempo, lo que hace la región operatoria menos sensible y la convalecencia más breve.

6. Se logra analgesia aumentando el umbral doloroso.

- Desventajas de la Analgesia Quirúrgica Acupuntural. ${ }^{42}$

1. El dolor no desaparece por completo. La analgesia acupuntural eleva el umbral del dolor, pero éste no desaparece por completo en todo momento ni en todas las personas.

2. La relajación muscular no es completa. La respuesta visceral interna no se puede controlar completamente. Durante las operaciones torácicas el paciente puede tener dificultad para la ventilación y a veces puede presentar fibrilación diafragmática. Durante las operaciones abdominales el enfermo puede experimentar sensaciones muy desagradables o dolor cuando se tira de las vísceras, lo cual lo irrita y le puede producir náuseas y vómitos. La relajación muscular no es satisfactoria.

3. El paciente consciente tiene que permanecer inmóvil. Como el operado está consciente y tiene que permanecer durante todo el acto quirúrgico en una misma posición, esto le puede ocasionar cansancio, molestias e intranquilidad.

4. Hay pacientes muy temerosos que no toleran ver el quirófano. Hay pacientes que, por temor a la operación, a la anestesia, a las agujas o simplemente al salón de operaciones, no admiten otra opción que no ver, oír ni sentir nada, y demandan la anestesia general o una sedación profunda.

5. La analgesia acupuntural no es efectiva en 10 a $15 \%$ de los pacientes. Independientemente de haberse realizado una buena selección y preparación del paciente y haber aplicado una técnica correcta, la AQA con relación a la anestesia convencional, resulta efectiva solo en 85 a $90 \%$. 
- Condiciones necesarias para realizar Analgesia Quirúrgica Acupuntural. ${ }^{43,44,42}$

1. Aplicarla en intervenciones de corta duración que no requieran presiones o tracciones fuertes ni relajación muscular.

2. Seleccionar pacientes con adecuada estabilidad psíquica, que estén de acuerdo con someterse a ser operados bajo esta técnica, que no teman a la operación ni al quirófano, a la acupuntura ni al estímulo eléctrico.

3. Garantizar una buena preparación preoperatoria del enfermo a fin de ganar su confianza y cooperación.

4. Disponer de las mejores condiciones para la operación: anestesiólogo acupunturista, cirujano de experiencia, diestro y con conocimientos de acupuntura, personal del quirófano que conozca la técnica acupuntural.

5. Seleccionar una técnica acupuntural adecuada: utilizar pocos puntos, solamente los indispensables, llevar a cabo la puntura con precisión y sin fallas, realizar una correcta estimulación de los puntos, con frecuencia e intensidad soportables por el paciente y durante el tiempo preciso.

6. Mantener una actitud optimista y segura, con una atención constante hacia el paciente que lo haga sentirse la persona más importante del equipo quirúrgico. Operar con rapidez y destreza, sin maniobras bruscas, y emplear correcta y oportunamente los medicamentos coadyuvantes a fin de evitar sufrimiento innecesario al enfermo.

7. Más que en una intervención quirúrgica bajo anestesia convencional, en la AQA el anestesiólogo debe realizar una premedicación correcta, tranquilizar al paciente con su atención directa y continua, controlar constantemente sus parámetros vitales y detectar y resolver de inmediato las alteraciones que se presenten durante la operación.

- $\quad$ Reglas en la selección de puntos para la analgesia acupuntural. ${ }^{43}$

- Puntos situados en el recorrido de los meridianos.

- Puntos situados en las concentraciones de conductos nerviosos. 
- Puntos situados en los músculos, cerca o a la altura del campo operatorio.

- Los puntos por debajo del nivel de los codos y de las rodillas producen una fuerte sensación “De Qi” y se utilizan en casi todas las operaciones.

- Los puntos auriculares Shenmen, simpático y pulmón, conjuntamente con otros puntos que corresponden a los campos operatorios.

- Contraindicaciones de la Analgesia Quirúrgica Acupuntural. ${ }^{42,43}$

1. Negación del paciente a ser operado con esta técnica.

2. Temor al quirófano, a la cirugía, a las agujas o a la corriente eléctrica.

3. Paciente que presente lipotimia durante la colocación de las agujas o la inducción de la analgesia.

4. Embarazadas y niños pequeños.

5. Intervención quirúrgica de larga duración, que necesite de relajación muscular total o que pudiera requerir algún tipo de ampliación.

6. Paciente que padezca de trastornos de la conducción eléctrica miocárdica o que posea marcapaso (contraindicación absoluta de la electroacupuntura).

7. Dolor o sangramiento al insertar las agujas; lipotimia por temor, ansiedad, cansancio, debilidad general, acupuntura dolorosa o estimulación incorrecta, agujas torcidas o rotas, neumotórax, lesión de órganos.

\section{Conclusiones}

La analgesia quirúrgica acupuntural no pretende ni puede sustituir de manera absoluta a la anestesia convencional, constituye una opción más, una alternativa a utilizar en determinados momentos y casos seleccionados, sola o como coadyuvante de distintas técnicas anestésicas.

Es innegable el efecto analgésico de la acupuntura, aunque todavía existen discrepancias con relación a sus mecanismos de acción, se ha avanzado en el esclarecimiento de los fundamentos 
científicos, teniendo en cuenta las diferentes teorías occidentales, lo que la hace más accesible a los profesionales de la salud, unido a su elevada efectividad en la práctica médica.

Existen numerosas razones para emplear la acupuntura en la actividad terapéutica diaria, sobre todo en el alivio del dolor, pero siempre que se realice con la seriedad, profundidad científica y el respeto que esta milenaria técnica se merece.

\section{Referencias bibliográficas}

1. Álvarez Díaz TA, Díaz Mastellari M. Manual de acupuntura.1992 [citado 11 enero 2017]. Disponible en: 〈http://bvs.sld.cu/libros/manual_deacupuntura/cap01.pdf>

2. Cabana Salazar J.A, Ruiz Reyes R. Analgesia por acupuntura. Rev Cubana Med Mil. 2004; 33(1): 10-15.

3. Din - Zongw. Acupunture and neurophysiology. Clin Neurosurg. 1990; 92 (1): 17-18.

4. Rigol Ricardo O. Manual de acupuntura y digitopuntura. La Habana: Editorial Ciencias Médicas; 1992.

5. Alvarez TO. Acupuntura. Medicina Tradicional Asiática. La Habana: Editorial Capitán San Luis; 1992.

6. Rigol Ricardo O. Apuntes para la historia de la acupuntura en Cuba. La Habana: sn; 1993.

7. González Pla EA, Perdomo Álvarez N, León Pujalte A. Generalidades sobre la analgesia quirúrgica acupuntural. MEDICIEGO. $2003 \quad$ [citado 23 Ene2016]; 9(2):49 Disponibleen:http://bvs.sld.cu/revistas/mciego/vol9_supl2_03/revisiones/r2_v9_supl203.htm.

8. Sánchez Valdeolla O E. La analgesia quirúrgica acupuntural como método anestésico. Arch Med. Camagüey. 2000; 4(1):48-53.

9. Benedetti C. Técnicas no farmacológicas de control del dolor agudo. La Habana: Ed Ciencias Médicas; 1987.

10. Pagola Bérger V. Analgesia Acupuntural Quirúrgica. Libro Digital. Librería virtual de Tlahui. México: Editorial Herbar. Biocyber Medicina Alternativa; 2003. 
11. Pérez F. Manual de acupuntura. La Habana: Editorial Ciencias Médicas; 1985.

12. Mirabal Mirabal ME, Sánchez Portela, CA, Oriolo Estrada MA, Martínez Zamora AL, García Rodríguez A. Uso de la analgesia acupuntural quirúrgica en la herniorrafía inguinal. Revista de Ciencias Médicas Pinar del Río. 2006; 10 (1)

13. Pagola Bérger V. Llovera Rodríguez JL, Pérez Borges N. La analgesia quirúrgica acupuntural en la cirugía del cuello y la cara. Hospital Universitario "Arnaldo Milián Castro". Villa Clara. 2006; 10 (4).

14. Abiague NicotC. Acupuncture analgesia in hand surgery. MEDISAN. 2010 [citado 2014 Jun 04]:14(5). Disponible en:

http://scielo.sld.cu/scielo.php?script=sci_arttext\&pid=S102930192010000500003\&lng=es.

15. Cabana Salazar JA, Ruiz Reyes R. Analgesia por acupuntura. Rev Cub Med Mil 2004 [citado 2014 Jun 04]; 33(1). Disponible en:

http://scielo.sld.cu/scielo.php?script=sci_arttext\&pid=S0138-65572004000100007\&lng=es.

16. Mulet Pérez A, Acosta Martínez B. Digitopuntura. Holguín: Ed Holguín; 1994.

17. Jun Z, Jing Z. Fundamentos de Acupuntura y Moxibustión de China. Editorial del

Instituto Latinoamericano de Medicina Oriental. 2009.

18. Stux G, Pomeranz B. Fundamentos de Acupuntura. 4ta ed. Barcelona. Springer- Verlag Ibérica. 2000.

19. Wang S, Kain Z, White P. Acupuncture Analgesia: I. the Scientific Basis. Anesth Analg, February 2008; 106 (2), 602-610.

20. Lim E. Acupuntura anestésica. Primera edición. Barcelona. Ediciones Ballaterra S.A. 2006.

21. Tiplt A, Bäumler PI, Irnich D. Acupuntura y dolor: una historia en tres partes. Rev Internacional de Acupuntura, 2010; 4(3).

22. Wang SM, Kain ZN, White PF. Acupuncture Analgesia: II. Clinical Considerations. The Scientific Basis. Anesth Analg, February 2008; 106(2): 611-621. 
23. Hecker H, Steveling et al. Acupuntura. Primera Edición. Colômbia. Manual Moderno. 2007.

24. Taquchi R. Acupuncture anesthesia and analgesia for clinical acute pain in Japan. CAM. 2008; 5(2), p153-8.

25. Conghuo T. Fundamentos de acupuntura y moxibustión de China. Pekín: Ediciones en Lenguas Extranjeras. 2003. p. V-VIII.

26. Ahn AC, Kaptchuk TJ. Advancing acupuncture research. Altern Ther Health Med. 2005; 11(3):40-5.

27. Chaitow L. Anestesia por acupuntura. Acupuntura y tratamiento del dolor. Barcelona: Editorial Bellaterra; 1988. p. 138-59.

28. Pagola Bérger V. Historia de la analgesia quirúrgica acupuntural. Analgesia Quirúrgica Acupuntural. México: Editorial Herbal, 2005. p. 20-4.

29. García A, Selin G. Analgesia quirúrgica acupuntural en pacientes geriátricos operados de catarata. Rev Misión Milagro. 2009; 1 (1).

30. Ni YF, Lian QQ, Jiang PW, Xu YQ. Application of acupuncture analgesia in colonoscopy. Chinese acupuncture \& moxibustion. 2007; 27(10): 766-8.

31. Bo QX, Zhang JX. Observation on therapeutic effect of scalp acupuncture analgesia on labor. Chinese acupuncture \& moxibustion. 2006 26(9):659-61.

32. Abreu Correa JM, Mateo JE. Analgesia acupuntural en las extracciones dentarias. Rev. Cubana Estomatol 1997; 34 (2): 10.

33. González Pla EA, Gómez Peire J, González Pacheco F. Analgesia quirúrgica acupuntural en cirugía ortopédica de la mano. MediCiego 2003; 9 (Sup 2): 49-54.

34. Uchitel OD. Transmisión sináptica. En: Tresguerres JAF. Fisiología Humana, 2da ed Madrid: McGraw-Hill, Interamericana, 1999. p. 48-71.

35. Zhang GG, Yu C, Lee W, Lao L, Ren K, Berman BM. Involvement of peripheral opioid mechanisms in electroacupuncture analgesia. NY. 2005; 1(5):365-71. 
36. Garrido Suárez B, Bosch Valdés F, Rabí Martínez MC, Hernández Arteaga M. Bases Neurobiológicas de la acupuntura y la electroacupuntura. Rev Hab C Médicas. 2004 3(10).

37. Harbach H, Moll B, Boedeker RH, Vigelius-Rauch U, OttoH, Muehling J, Hempelmann G, Markart P. Minimal immunoreactive plasma beta-endorphin and decrease of cortisol at standard analgesia or different acupuncture techniques. Eur J Anaesthesiol. 2007; 24(4):370-6.

38. He D, Hostmark AT, Veiersted KB, Medbo JI. Effect of intensive acupuncture on painrelated social and psychological variables for women with chronic neck and shoulder pain--an RCT with six month and three year follow up. Acupunct Med. 2005; 23(2): 52-61.

39. Kong J, Fufa DT, Gerber AJ etal. Psychophysical outcomes from a randomizad pilot study of manual, electro and sham acupuncture treatment on experimentally induced thermal pain. J Pain .2005; 6(1): 55-64.

40. Reisine T, PasternakG. Analgésicos opioides y sus antagonistas. Las Bases Farmacológicas de la Terapéutica. 10ma ed. México: Editorial Mc Graw-Hill Interamericana; 2002. p. 557-594.

41. Mendoza Rojo C, Cantera López B. Bases neurofisiológicas, neuroquímicas y cognoscitivas del dolor. Efectos de la acupuntura y la electroacupuntura. Reflexoterapia /S.N/, 1992: 1-20.

42. Pagola Bérger V. Características de la A.Q.A. En: Analgesia Quirúrgica Acupuntural. México: Editorial Herbal, 2005. p. 42-48.

43. Pagola Bérger V. Preparación para la A.Q.A. En: Analgesia Quirúrgica Acupuntural. México: Editorial Herbal, 2005. p. 48-62.

44. Ramos Portuondo H. Colonoscopía con analgesia quirúrgica acupuntural. MEDISAN 2016; 20(11): 5129 . 